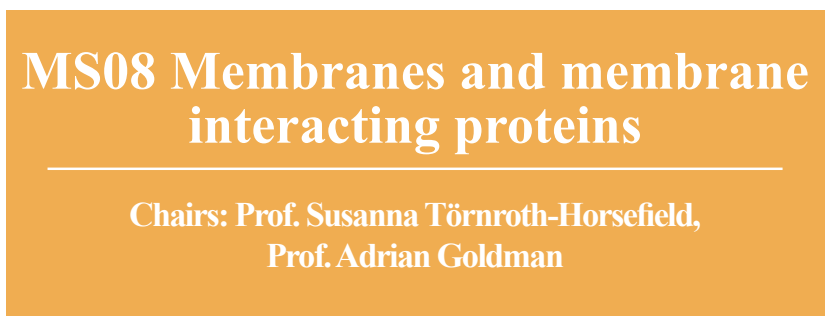

MS08-01

\section{P-type ATPase inhibitors: something old, something new, leads to follow and some new clues}

Maike Bublitz ${ }^{1}$

1, Department of Biochemistry, University of Oxford, Oxford, United Kingdom

email: maike.bublitz@bioch.ox.ac.uk

P-type ATPases are a widespread family of membrane pumps that mediate a plethora of essential cellular transport functions. Consequently, many P-type ATPases rank as prime drug targets, both in the context of human disorders and infectious diseases. The exploration of potent P-type ATPase inhibitors throughout the last decades has in turn strongly advanced our understanding of the molecular pumping mechanism that underlies substrate transport across lipid bilayers. One of the most widely used inhibitors in P-type ATPase research is vanadate, although structural details of its inhibitory mechanism have long remained unresolved. We have determined the crystal structure of the sarcoplasmic reticulum $\mathrm{Ca}^{2+}$-ATPase (SERCA) in complex with vanadate [1], allowing a description of its binding mode in the catalytic site, along with some novel insight into ion and nucleotide binding sites in SERCA. In addition to the phosphate-mimic vanadate, we have further identified a novel class of P-type ATPases inhibitors [2]. Using a set of rationally designed compounds, we have analyzed their structure-activity relationship by functional assays, crystallography and computational modeling. These new inhibitors are candidates for further development towards drugs against invasive fungal infections.

References:

[1] Bublitz, M. et al. (2018). Tetrahydrocarbazoles are a novel class of potent P-type ATPase inhibitors with antifungal activity. PLoS One 13:e188620.

[2] Clausen*, J. D., Bublitz*, M. et al. (2016). Crystal Structure of the Vanadate-Inhibited Ca2+-ATPase. Structure 24:617-623. *contributed equally

Keywords: P-type ATPases, Membrane pumps, Inhibitory compounds

\section{MS08-02}

\section{Membrane transport by proteins of the $7+7$ inverted repeat superfamily: variation on a theme}

\author{
Alexander Cameron ${ }^{1}$ \\ 1. University of Warwick, Coventry, United Kingdom \\ email: a.cameron@warwick.ac.uk
}

Secondary active membrane transport proteins specifically move molecules across membranes. As first posited by Jardesky in the 1960 s, they work by the alternating access mechanism, in which the binding site of the protein is open alternately to one side of the membrane and then, through a conformational change, to the other. This enables the molecule to be carried across the membrane. Membrane transporters have been divided into families based on sequence and substrate specificity. Over the last decade many structures have been solved of proteins from different families. Through these studies it has been seen that, though the topology of the proteins varies, they often contain inverted repeats and these repeats have been implicated in the transport mechanism. Two of the more recent structures we solved, the human anion exchanger $1^{1}$ and the uric acid/xanthine $\mathrm{H}(+)$ symporter, UapA from Aspergillus nidulans ${ }^{2}$, both have a $7+7$ inverted repeat. I will discuss the mechanisms of these two proteins and how they compare to other membrane transport proteins that we have solved previously.

\section{References:}

[1] Arakawa, T., Kobayashi-Yurugi, T., Alguel, Y., Iwanari, H., Hatae, H., Iwata, M., Abe, Y., Hino, T., Ikeda-Suno, C., Kuma, H., Kang, D., Murata, T., Hamakubo, T., Cameron, A. D., Kobayashi, T., Hamasaki, N. \& Iwata, S. (2015). Science. 350, 680-4.

[2] Alguel, Y., Amillis, S., Leung, J., Lambrinidis, G., Capaldi, S., Scull, N. J., Craven, G., Iwata, S., Armstrong, A., Mikros, E., Diallinas, G., Cameron, A. D. \& Byrne, B. (2016). Nat Commun. 7, 11336.

Keywords: Membrane transport proteins, mechanism 